\title{
Temporal relationships of lumbar herniated disk injuries
}

\author{
BRIAN F. MCCRARY, DO
}

To evaluate the relationship of time of day to severe low-back injuries, 60 cases of herniated nucleus pulposus were selected from five occupational medicine clinics. Causes of injury were assigned to three categories (material handling, body reactions, and falls), and the actual time of injury was recorded. A significant association was noted between nonfalling injuries and passage of time on workshift $(P<.025)$. This pattern was consistent for both male and female patients and for clinic sites.

Low-back pain is one of the most common medical conditions in the Western world; it afflicts approximately $85 \%$ of all persons sometime during their lives. ${ }^{1}$ Annually, $2 \%$ of the national work force incurs industrially related back injuries. ${ }^{1}$ In fact, low-back pain is the leading compensable workrelated injury and the second most common cause of absence from work. ${ }^{1}$

In 1984 , at least $\$ 16$ billion was expended in the United States on the treatment of low-back pain and compensation payments associated with this condition. Incidence rates do not explain adequately the economic impact of workers with low-back injuries. The small percentage of claimants who receive permanent total or partial disability payments are responsible for a disproportionate amount of the total costs associated with back pain or injury. ${ }^{3}$ One group of investigators (S.H. Snook, M.D., R.A. Campanelli, M.D., R.J. Ford, M.D., Liberty Mutual Insurance Company, Hopkinton, Mass, 1980 ) found that $25 \%$ of cases account for $90 \%$ of the cost. A significant proportion of these severe, prolonged injuries are attributable to the herniated lumbar intervertebral disk. ${ }^{4}$

Reducing the cost of back trauma in industry depends to a large degree on controlling or pre- venting the small percentage of high-cost injuries. Many investigators have centered their attention on injury factors, particularly on those activities and events associated with the onset of symptoms. Various studies ${ }^{5-11}$ have concentrated on workplace, individual, and social and psychologic factors to determine significant associations.

Bigos and associates ${ }^{1}$ examined appraisal ratings, years of service, and job skill, along with day of the week, month, and workshift when the injury occurred. They concluded that a significantly higher proportion of high-cost injuries were noted during the day than on evening or night shifts. They did not attempt to examine this finding further.

No published study to date has examined the hour of injury to determine whether a significant proportion of injuries occur early or later in the typical eight-hour workshift. Theoretically, it would be expected that an increased proportion on injuries would occur in the later hours of the working shift, because of structural fatigue and changes in disk composition as erect time lengthens, thus making the disk more susceptible to injury. ${ }^{12-18}$ In addition to these changes in the disk itself, fatigue of the spinal structures is related to dynamic stress raised to a power of 10 . This means that a reduction in strength or an increase in dynamic load has a disproportionately large effect on weak spinal tissues, or for those exposed to high-dynamic loads. ${ }^{17}$

Therefore, in the present study, I examined records of patients who had been diagnosed with herniated nucleus pulposus injuries to determine whether time during the workshift is a significant factor for severe low-back injuries.

\section{Methods}

Workman's Compensation records from five different occupational medicine clinics in Tucson and Phoenix for the years 1977 to 1986 were examined. 
All high-cost $(\geqslant \$ 500)$ or long-duration $(\geqslant$ three weeks) cases of low-back injury were analyzed.

A total of 60 cases of diagnosed lumbar herniated nucleus pulposus were identified. (Cases involving the disk descriptions "ruptured," "free fragments," "herniated," "prolapsed," "bulging," and "extruded" are included, but not disk degeneration without evidence of nerve root involvement.) Thirtyone cases were obtained from four independent occupational medicine clinics, and 29 were obtained from a fifth clinic, which had a disproportionate number of severe low-back injury cases. This clinic performed a large number of disability evaluations per year, with many of these patients being referred after initial treatment in other offices and clinics.

The population at risk, which consisted of all workers presenting to any of these five clinics for initial treatment of their injury, included craftsmen, laborers, medical personnel, drivers, managers, clerks, and teachers. Patients older than 58 years were eliminated from the study because of the increasing frequency with age of osteoporosis, neoplasms, and associated medical conditions.

The diagnosis of herniated nucleus pulposus was confirmed in each case by myelography, computed tomography, or magnetic resonance imaging, or by surgery (where the surgeon stated on the chart that the herniated disk was present during surgery and the patient had symptoms of radicular pain along the sciatic nerve distribution).

Cases were excluded when a previous diagnosis of herniated nucleus pulposus, lumbar spondylolisthesis, anklylosing spondylitis, spinal stenosis, or lumbar fracture had been recorded, or when the time of injury had not been noted on the incident report or elsewhere in the chart.

Only patients working a daytime eight-hour workshift (for example, $8 \mathrm{AM}$ to $5 \mathrm{PM}, 7 \mathrm{AM}$ to $4 \mathrm{PM}$ ) were included. For each patient, the following information was recorded: age, sex, prior history of low-back injury examined by a physician, cause of

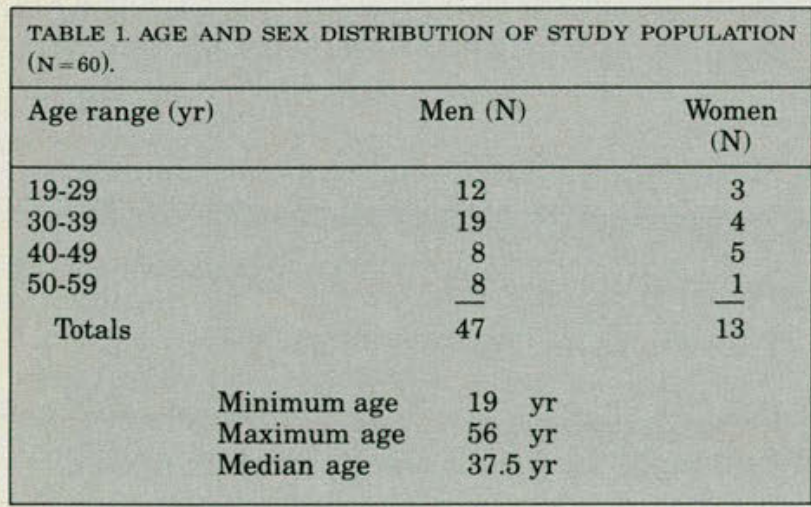

injury, normal workshift hours, date, and time of injury.

Causes of injury were assigned to one of three categories - material handling, body reactions, or falls. Material handling injuries were incurred while lifting, pushing, pulling, carrying, or tying loads. Body reaction injuries were caused by slips, twisting, or direct blows, or by working in cramped positions. In the third category, a direct fall onto the back or hip resulted in injury.

Time of injury was assigned to one of four quartiles for descriptive purposes. Each quartile represented two hours of the total eight-hour work time. For example, an employee who worked from $8 \mathrm{AM}$ to $5 \mathrm{PM}$, with a one-hour lunch break, was placed in the second quartile if his injury occurred at 10:30 $\mathrm{AM}$, and in the third quartile if it happened at 1:00 PM.

\section{Results}

The age and sex distribution of the study population is shown in Table 1. Temporal analysis of the 60 cases of herniated nucleus pulposus injuries is presented in Fig 1. The relationship with time is nearly linear, although the sample size is too small to show statistical significance.

The number and percentage of patients with a documented prior history of low-back problems evaluated by a physician is presented in Table 2 . Thirty-eight patients $(63 \%)$ had at least one posi-

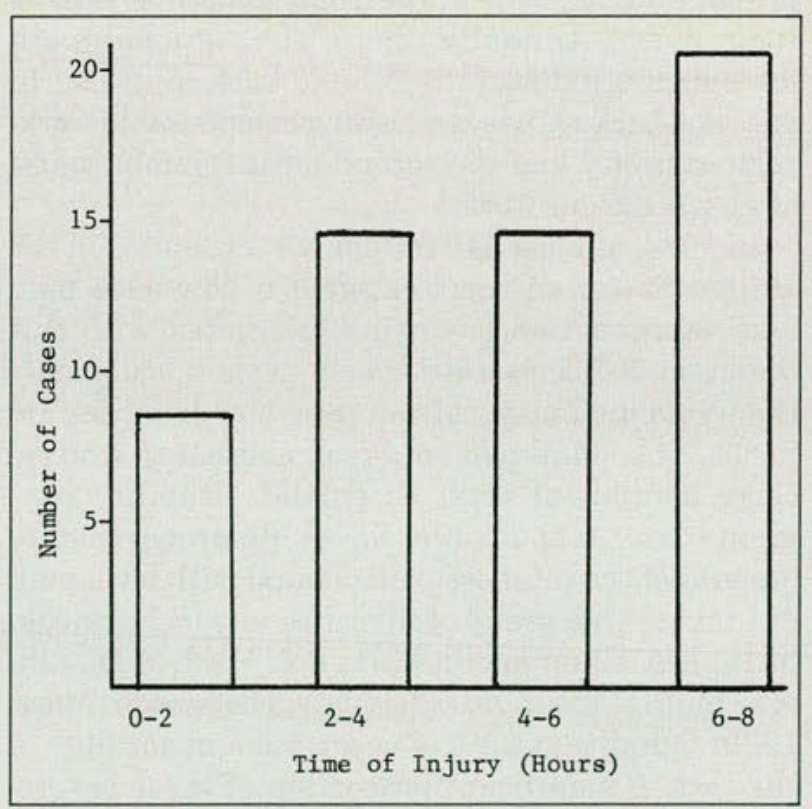

Fig 1. Cases of severe low-back injuries compared to amount of time elapsed on day shift ( $0-2$ hours, $N=9 ; 2-4$ hours, $N=15$; 4-6 hours, $N=15$; $6-8$ hours, $N=21$ ). 


\begin{tabular}{|lc|}
\hline TABLE 2. PAST HISTORY OF LOW-BACK PROBLEMS $(\mathrm{N}=60)$. \\
\hline History & $\mathrm{N}$ \\
\hline Positive & 38 \\
Negative & 15 \\
Unknown & 7 \\
\hline
\end{tabular}

TABLE 3. DISTRIBUTION BY CAUSE OF INJURY AND TIME ELAPSED ON DAY SHIFT $(\mathrm{N}=60)$.

\begin{tabular}{|lrrrc|}
\hline & \multicolumn{4}{c|}{ Time of injury (hr) } \\
\cline { 2 - 5 } Cause & $0-2$ & $2-4$ & $4-6$ & $6-8$ \\
\hline Falls & 2 & 9 & 1 & 5 \\
Material handling & 5 & 6 & 8 & 9 \\
Body reactions & $\underline{2}$ & $\underline{0}$ & $\underline{6}$ & $\underline{7}$ \\
Totals & 9 & 15 & 15 & 21 \\
\hline
\end{tabular}

tive documented episode of low-back pain. All had been released by their physicians for full duty with no restrictions. Fifteen (25\%) specifically denied any prior history of low-back problems. Seven $(12 \%)$ did not specify whether low-back injury had occurred in the past. On chi square analysis of these data, there is no significant association between prior history and cause or timing of injury.

Documented causes of these injuries are shown in Table 3. The overall temporal pattern of injuries occurring later in the workshift is consistent with material handling and body reaction types of injuries, but it is reversed with injuries caused by falls. Most shifts were organized with four hours of work, a lunch break, then four more hours of work, followed by a terminal break. The data suggested that falls tended to occur before major breaks (Table 3), but larger sample sizes would be needed to confirm this association. There was a significant difference between falls and nonfalling injuries when compared by passage of time on the shift $\left(\mathrm{X}^{2}=4.36, P<.05\right)$.

When rates of nonfalling accidents are compared between first and second halves of the shifts, the association between time and injury is significant $\left(\mathrm{X}^{2}=5.76, P<.025\right)$. There is no difference in this association when sex is considered (Table 4). The same pattern was present in each age group and at each clinic site, although the sample sizes are too small to show statistical significance.

\section{Conclusions}

Clearly, low-back injuries are one of the most frquent and disabling conditions affecting people during their productive years. Further, a small percentage of these injuries incur a disproportionate share of costs. A significant proportion of severe, prolonged cases are attributable to the herniated

\begin{tabular}{|c|c|c|}
\hline & Time & (hr) \\
\hline Sex & $0-4$ & $4-8$ \\
\hline Female & 4 & 9 \\
\hline Male & $\underline{20}$ & $\underline{27}$ \\
\hline Totals & $\overline{24}$ & $\overline{36}$ \\
\hline
\end{tabular}

lumbar intervertebral disk. Workplace factors associated with these injuries must be identified if we are to concentrate on prevention where it is most needed.

Low-back injuries, especially those that are not related to falling, occur more frequently in the later hours of the workshift. This pattern is seen among all age groups, in both sexes, and at all clinic sites examined. This study focused on the high-cost cases. Further research could determine whether all low-back injury cases have this same temporal pattern.

I hypothesize that fatigue of the structural elements of the spine or even breakdown in these structures is occurring as a result of dynamic loading, torsioning, bending, vibration, or other activity that stresses these structures throughout the day. Numerous study ${ }^{12-18}$ results support the idea that these structural changes are occurring.

In this case, perhaps incorporation of rest periods later in the workshift, along with frequent postural changes, could lead to a decrease in severe injuries. Also, increased supervision and greater attention to proper safety techniques and methods of material handling in the later hours of the workshift potentially could lead to long-term savings.

Another suggestion for research is quantification of the load handled to determine the importance of this variable. This was not possible in this retrospective, chart-review type of study.

1. Bigos SJ, Spengler DM, Martin NA, et al: Back injuries in industry, a retrospective study: Part I. Overview and cost analysis. Part III. Employee-related factors. Spine 1986;11:241-245;252-256.

2. Grazier KL, Holbrook TL, and Kelsey JL, et al. The frequency of occurrence, impact, and cost of musculoskeletal conditions in the United States. Chicago, American Academy of Orthopedic Surgeons, 1984.

3. Leavitt SS, Johnston TL, Beyer RD: The process of recovery: Patterns in industrial back injuries. Ind Med 1972;41:7-11.

4. Benn RT, Wood PHN: Pain in the back: An attempt to estimate the size of the problem. Rheumatol Rehab 1975;14:121-128.

5. Frymoyer JW, Pope MH, Costanza MC, et al: Epidemiologic studies of low back pain. Spine 1980;5:419-423.

6. Kelsey JL: An epidemiological study of acute herniated lumbar intervertebral disks. Rheumatol Rehab 1975;14:144-158.

7. Kelsey JL: An epidemiological study of the relationship between occupations and acute herniated lumbar intervertebral disks. Int $J$ Epidemiol 1975;4:197-204. 
8. Magora A: Investigation of the relationship between low back pain and occupation: Parts 1 and 2. Ind Med Surg 1970;39:465-471,504-510.

9. Magora A: Investigation of the relations between low-back pain and occupation: Part 3. Physical requirements. Fittings, standing, and weightlifting. Ind Med Surg 1972;41:5-9.

10. Rowe ML: Low back pain in industry: A position paper. $J$ Occup Med 1979;11:161-169.

11. Westrin CG: Low back sick-listing: A nosological and medical insurance investigation. Acta $J$ Soc Med Scand 1970;2:127-134.

12. Adams MA, Hutton, WC: The effect of posture on the fluid content of lumbar intervertebral disks. Spine 1983;8:665-671.

13. Chaffin DB, Park KS: A longitudinal study of low back pain as associated with occupational weight lifting factors. Am Ind Hyg Assoc J 1973;34:513-525.

14. Eklund JA, Corlett EN: Shrinkage as a measure of the effect of load on the spine. Spine 1984;9:189-194.
15. Liu YK, Goel VK, DeJong A, et al: Torsional fatigue of the lumbar intervertebral joints. Spine 1985;10:894-900.

16. Markolf KL, Morris JM: The structural components of the intervertebral disk. J Bone Joint Surg 1974;56A:675-687.

17. Sandover J: Dynamic loading as a possible source of low-back disorders. Spine 1983;8:652-658.

18. Wilder DG., Woodworth BB, Frymoyer JW, et al: Vibration and the human spine. Spine 1982;7:243-254.

From the Department of Family and Community Medicine, University of Arizona Health Services Center, Tucson.

Reprint requests to Dr McCrary, PO Box 27903, Tucson, AZ 85726. 


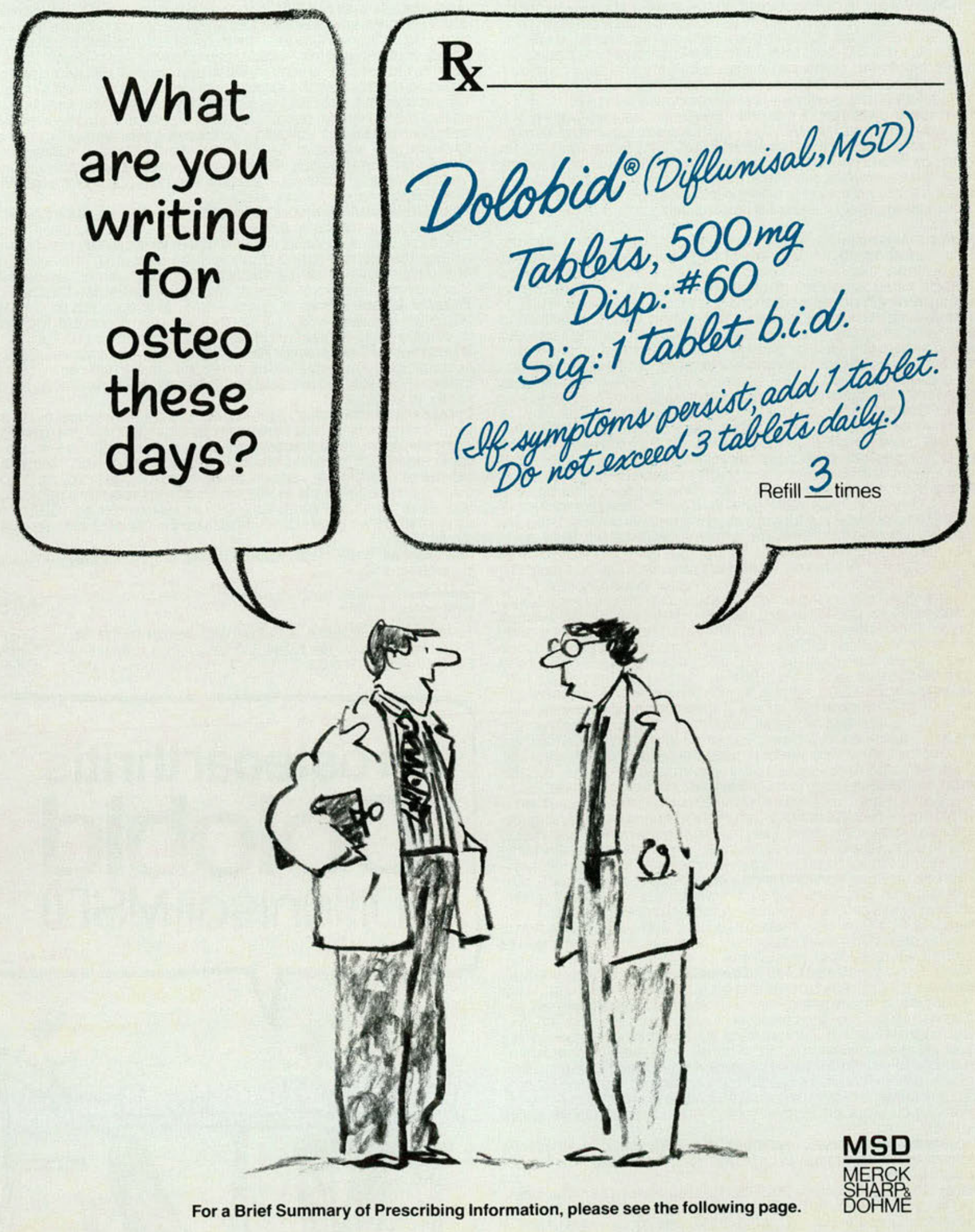


Contraindications: Hypersensitivity to this product; patients in whom acute asthmatic attacks, urticaria, or rhinitis is precipitated by aspirin or other nonsteroidal anti-inflammatory drugs.

Warnings: Peptic ulceration and gastrointestinal bleeding have been reported. Fatalities have occurred rarely. Gastrointestinal bleeding is associated with higher morbidity and mortality in patients acutely ill with other conditions, the elderly, and patients with hemorrhagic disorders. In patients with active gastrointestinal bleeding or an active peptic ulcer, benefits 0 therapy with DOLOBID ${ }^{\circledast}$ (Diflunisal, MSD) must be weighed against possible hazards, an appropriate ulcer regimen should be instituted, and the patient's progress carefully monitored: in patients with a history of upper or lower gastrointestinal tract disease, DOLOBID should be given only after consulting the Adverse Reactions section and under close supervision.

Precautions: General: Although DOLOBID has less effect on platelet function and bleeding time than aspirin, at higher doses it is an inhibitor of platelet function; patients who may be adversely affected should be carefully observed. Because of reports of adverse eye findings with agents of this class, it is recommended that patients who develop eye complaints during treatment have ophthalmologic studies. Peripheral edema has been observed; as with othe drugs in this class, DOLOBID should be used with caution in patients with compromised cardiac function, hypertension, or other conditions predisposing to fluid retention. Acetylsalicy lic acid has been associated with Reye syndrome; because diffunisal is a derivative of salicylic acid, the possibility of its association with Reye syndrome cannot be excluded

Hypersensitivity Syndrome: A potentially life-threatening, apparent hypersensitivity syndrome has been reported. This multisystem syndrome includes constitutional symptoms (fever, chills), and cutaneous findings (see ADVERSE REACTIONS, Dermatologic), and may also include involvement of major organs (changes in liver function, jaundice, leukopenia, thrombocytopenia, eosinophilia, disseminated intravascular coaqulation, renal impairment, including renal failure), and less specific findings (adenitis, arthralgia, myalgia, arthritis, malaise, anorexia, disorientation). If evidence of hypersensitivity occurs, DOLOBID should be discontinued.

Renal Effects: As with other nonsteroidal anti-inflammatory drugs, long-term administration of diflunisal to animals has resulted in renal papillary necrosis and other abnormal renal pathology: In humans, there have been reports of acute interstitial nephritis with hematuria and proteinuria and occasionally nephrotic syndrome. A second form of renal toxicity has been seen in patients with prerenal and renal conditions leading to a reduction in renal blood flow or blood volume, where renal prostaglandins have a supportive role in the maintenance of renal perfusion. In these patients, administration of an NSAID may cause a dose-dependent reduction in prostaglandin formation and may precipitate overt renal decompensation. Patients at greatest risk of this reaction are those with conditions such as renal or hepatic dysfunction, diabetes mellitus, advanced age, extracellular volume depletion from any cause congestive heart failure, septicemia, pyelonephritis, or concomitant use of any nephrotoxic drug. DOLOBID or other NSAIDs should be given with caution and renal function should be monitored in any patient who may have reduced renal reserve. Discontinuation of NSAID therapy is typically followed by recovery to the pretreatment state. Since diflunisal is eliminated primarily by the kidneys, patients with significantly impaired renal function should be closely monitored; a lower daily dosage should be anticipated to avoid excessive drug accumulation. Laboratory Tests: As with other nonsteroidal anti-inflammatory drugs, borderline elevations of one or more liver tests may occur in up to $15 \%$ of patients. These abnormalities may progress, may remain essentially unchanged, or may be transient with continued therapy. The SGPT (ALT) test is probably the most sensitive indicator of liver dysfunction. Meaningful ( 3 times the upper limit of normal) elevations of SGPT or SGOT (AST) occurred in controlled clinical trials in less than $1 \%$ of patients. A patient with symptoms and/or signs suggesting liver dysfunction, or in whom an abnormal liver test has occurred, should be evaluated for evidence of the development of more severe hepatic reactions while on therapy with DOLOBID Severe hepatic reactions, including jaundice, have been reported with DOLOBID as well as with other nonsteroidal anti-inflammatory drugs. Although such reactions are rare, if abnormal liver tests persist or worsen, if clinical signs and symptoms consistent with liver disease develop, or if systemic manifestations occur (e.g. eosinophilia, rash, etc.), DOLOBID should De discontinued since liver reactions can be fatal.

Drug Interactions: DOLOBID prolongs prothrombin time in patients who are on oral anticoagulants. It has not been shown to interact with tolbutamide but interacts with hydrochlorothiazide, furosemide, acetaminophen, aspirin, indomethacin, sulindac, and naproxen Ora Anticoagulants - In some normal volunteers, concomitant administration of DOLOBID and warfarin, acenocoumarol, or phenprocoumon resulted in prolongation of prothrombin time. Accordingly prothrombin time should be closely monitored during and for several days after concomitant administration; dosage of oral anticoagulants may require adjustment. Tolbuamide - In diabetic patients, no significant effects were seen on tolbutamide plasma levels or fasting blood glucose. Hydrochlorothiazide-In normal volunteers, concomitant administraion significantly increased the plasma levels and decreased the hyperuricemic effect of hydrochlorothiazide. Furosemide - In normal volunteers, concomitant administration had no effect on the diuretic activity but decreased the hyperuricemic effect of furosemide. Antacids Concomitant administration may reduce plasma levels of DOLOBID; this effect may be clinically significant when antacids are used on a continuous schedule. AcetaminophenConcomitant administration in normal volunteers increased plasma levels of acetaminophen approximately $50 \%$ but had no effect on plasma levels of DOLOBID. Since acetaminophen in high doses has been associated with hepatotoxicity, concomitant administration should be

Drug Interactions: Nonsteroidal Anti-inflammatory Drugs: In normal volunteers on indomethacin, diflunisal decreased the renal clearance and significantly increased plasma levels of indomethacin; in some patients, such combined use was associated with fatal gastrointestinal hemorrhage. These drugs should not be used concomitantly. Safety and effectiveness of concomitant use with other nonsteroidal anti-inflammatory drugs have not been established, and no recommendation for concomitant use can be made. The following information was obtained from studies in normal volunteers: Aspirin -A small decrease in diflunisal levels with multiple concomitant doses: Sulindac-A lowering of plasma levels of active sulindac sulfide metabolite by approximately one third: Naproxen - No effect on plasma levels of either drug, but significantly decreased urinary excretion of naproxen and its glucuronide metabolite.

Carcinogenesis, Mutagenesis, Impairment of Fertility: Diflunisal did not show carcinogenic potential in long-term studies in rats and mice. It had no mutagenic activity, and did not impair fertility.

Pregnancy: Pregnancy Category C-DOLOBID should be used during the first two trimesters of pregnancy only if the potential benefit justifies the potential risk to the fetus; because of the known effect of drugs of this class on the human fetal cardiovascular system (closure of ductus arteriosus), use during the third trimester is not recommended

Nursing Mothers: Diflunisal is excreted in human milk in concentrations of $2 \%$ to $7 \%$ of those in plasma. A decision should be made whether to discontinue nursing or to discontinue the drug, taking into account the importance of the drug to the mother.

Pediatric Use: Safety and effectiveness in infants and children have not been established, and use in children below 12 years of age is not recommended.

Adverse Reactions: The controlled clinical trials encompass observations in 2.427 patients. Listed below are the adverse reactions reported in the 1,314 patients who received treatment in studies of two weeks or longer Of these patients, 513 were treated for at least 24 weeks, 255 for at least 48 weeks, and 46 for 96 weeks. In general, the adverse reactions were 2 to 14 times less frequent in the 1,113 patients who received short-term treatment for mild to moderate pain. The most frequent types of adverse reactions occurring are gastrointestinal. Incidence Greater than 1\%: Gastrointestinal - Nausea,* vomiting, dyspepsia,* gastrointestinal pain,* diarrhea,* constipation, flatulence. Psychiatric - Somnolence, insomnia. Central Nervous System-Diziness. Special Senses-Tinnitus. Dermatologic-Rash.* Miscellaneous-Headache* fatique/tiredness.

Incidence Less than 1 in 100: The probability exists of a causal relationship between DOLOBID and these adverse reactions. Dermatologic - Erythema multiforme, exfoliative dermatitis, Stevens-Johnson syndrome, toxic epidermal necrolysis, urticaria, pruritus, sweating, dry mucous membranes, stomatitis, photosensitivity. Gastrointestinal-Peptic ulcer, gastrointestinal bleeding, anorexia, eructation, gastrointestinal perforation, gastritis; liver function abnormalities; jaundice, sometimes with fever; cholestasis; hepatitis. Hematologic - Thrombocytopenia, agranulocytosis. Genitourinary - Dysuria; renal impairment, including renal failure; interstitial nephritis; hematuria; proteinuria. Psychiatric-Nervousness, depression, hallucinations, confusion, disorientation. Central Nervous System-Vertigo, light-headedness, paresthesias. Special Senses-Transient visual disturbances including blurred vision. Hypersensitivity Reactions - Acute anaphylactic reaction with bronchospasm; angioedema. Hypersensitivity vasculitis. Hypersensitivity syndrome (see PRECAU-

\section{TIONS). Miscellaneous Asthenia, edema.}

Causal Relationship Unknown: Other reactions have been reported in clinical trials or since the drug was marketed but occurred under circumstances where a causal relationship could not be established. However, in these rarely reported events, that possibility cannot be excluded. Therefore, these observations are listed to serve as alerting information to physicians. Respiratory-Dyspnea. Cardiovascular-Palpitation, syncope. MusculoskeletalMuscle cramps. Genitourinary-Nephrotic syndrome. Miscellaneous - Chest pain

Potential Adverse Effects: In addition, a variety of adverse effects not observed with DOLOBID* (Diflunisal, MSD), but reported with other nonsteroidal analgesic/antiinflammatory agents, should be considered potential adverse effects of DOLOBID.

Management of Overdosage: In the event of overdosage, the stomach should be emptied by inducing vomiting or by gastric lavage, and the patient carefully observed and given symptomatic and supportive treatment. Because of the high degree of protein binding, hemodialysis may not be effective

Dosage and Administration: For mild to moderate pain an initial dose of $1000 \mathrm{mg} f \mathrm{l}-$ lowed by $500 \mathrm{mg}$ every 8-12 hours is recommended for most patients. A lower dosage may be appropriate depending on such factors as pain severity, patient response, weight, or advanced age: for example, $500 \mathrm{mg}$ initially, followed by $250 \mathrm{mg}$ every 8-12 hours. For osteoarthritis and rheumatoid arthritis, the suggested dosage range is $500 \mathrm{mg}$ to $1000 \mathrm{mg}$ daily in two divided doses; the dosage may be increased or decreased according to patient response. Maintenance doses higher than $1500 \mathrm{mg}$ a day are not recommended. DOLOBID may be administered with water, milk or meals. Tablets should be swallowed whole, not crushed or chewed

How Supplied: Tablets, containing 250 or $500 \mathrm{mg}$, in unit-dose packages of 100 and unitof-use bottles of 60

Incidence between $3 \%$ and $9 \%$. Those reactions occurring in $1 \%$ to $3 \%$

For more detailed information, consult your MSD Representative or see Prescribing Information. Merck Sharp \& Dohme, Division of Merck \& Co. lve. West Point, PA 19486

J7DL33R2(723)

MSD MERCK SHARP
DOHME

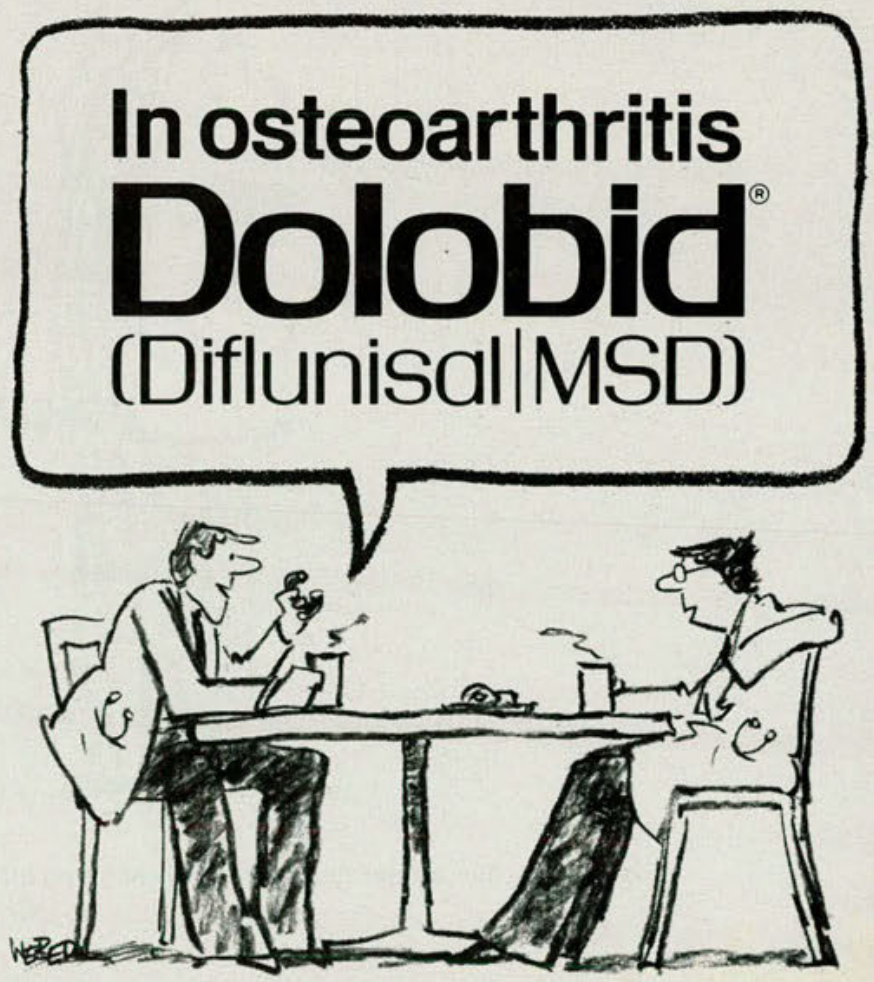

